\title{
Review on Volatile Flavor Components of Roasted Oilseeds and Their Products
}

\section{Cuicui, HOU Lixia ${ }^{*}$}

\author{
College of Food Science and Technology, Henan University of Technology, Zhengzhou 450001, China
}

\begin{abstract}
Volatile flavor components in foods are the key quality parameters. The researchers conducted in-depth research on sesame oil, peanut oil, rapeseed oil, sunflower oil, sesame paste and peanut butter, etc. However, there is no comprehensive summary about the flavor of roasted oilseeds and their products. The occurrence and the formation mechanisms of the main volatile flavor substances, and the impacts of processing factors are discussed in this review, which may provide references for further research on volatile flavor compounds, identification and evaluation of specific products and development of new products.
\end{abstract}

Keywords: Roasted Oilseeds and their products; Volatile compounds; Formation

\section{Introduction}

Vegetable oilseeds and their products are rich in oil, protein and vitamins. The flavor is one of the most important indexes to measure the quality of food. The purposes of this review are to compile occurrence and odor note of the main volatile flavor substances present in the vegetable oilseeds and their products, discuss the proposed formation mechanism, and outline the impact of processing factors. The study of volatile flavor components in roasted oilseeds and their products is of great significance to guide the processing, and promote the development of oilseed products.

\section{The Main Volatile Compounds in Roasted Oilseeds and Their Products}

The researches have showed that the volatile compounds of roasted oilseeds and their products mainly include pyrazine, furan, aldehydes, alcohols, ketones, acids, lipids, sulfur compounds, alkenes, alkanes ${ }^{[1-5]}$, etc.

\subsection{Pyrazines}

Pyrazines are main volatile flavor components in roasted oilseeds and their products, usually with nutty and roast flavor ${ }^{[6]}$, have lower flavor thresholds. According to the study, pyrazines are the main flavor substances of roasted sesame seeds, roasted sunflower seeds, sesame oil, peanut oil, aromatic sunflower oil, peanut butter and sesame paste ${ }^{[7-12]}$. When the roasting temperature is above $170{ }^{\circ} \mathrm{C}$, the main volatiles of hot-pressed sunflower seed oil and perilla seed oil are the heterocyclic substances, such as pyrazine ${ }^{[5,13]}$.

About 19 pyrazines have been reported in oilseeds and their products $^{[7-16]}$, including 2,5-dimethylpyrazine, 2,3-dimethylpyrazine, 2,3,5-trimethyl pyrazine, 3-ethyl-2,5-dimethylpyrazine, 2-acetyl

Received: 7 September 2018 /Accepted: 4 December 2018.

Supported by Modern Agricultural Industrial Technology System Construction Project(CARS14-1-29).

*Corresponding author. E-mail: hou1269@126.com

(CHenan University of Technology 2018

LI C C, HOU L X. Review on Volatile Flavor Components of Roasted Oilseeds and Their Products[J]. Grain \& Oil Science and Technology, 2018, 1(4): 151-156. -3-methylpyrazine ${ }^{[10]}, 2$-ethyl-5-methylpyrazine, 2-ethyl-6-methyl pyrazine ${ }^{[6]}$, and so on. Among them, 2,5-dimethylpyrazine is one of the main volatile components of roasted peanut, peanut oil, sunflower oil and rapeseed oil ${ }^{[10-11,14,16]}$. In addition, the key flavor compound for roasted peanuts is ethyl pyrazine ${ }^{[17]}$, and the sesame oil have the higher levels of 2-methylpyrazine, and 2,6-dimethylpyrazine ${ }^{[18]}$. Furthermore, methylpyrazine and 5H-5-Methanol-6,7-dihydrocyclopentapyrazine are the important reference for the evaluation of the flavor quality of sesame oil ${ }^{[15]}$. The most important pyrazine in rapeseed oil produced from roasted seeds are 2,3-diethyl-5-methylpyrazine and 3-isopropyl-2-methoxy Pyrazine ${ }^{[4]}$.

\subsection{Aldehydes}

Aldehydes, with low threshold values, are commonly found in the roasted oilseeds and their products, and mainly contribute to the overall odor of the roasted oilseeds and their products ${ }^{[19]}$. They mainly come from the oxidation and degradation of oils and the degradation of amino acids in Strecker reaction, which is related to the bad flavor of oils ${ }^{[2,6]}$. Aldehydes are considered to the key volatile compounds in roasted peanuts, virgin olive oil, rapeseed oil, camellia oil and sesame paste ${ }^{[8,17,20-22]}$.

There are about 14 aldehyde compounds reported ${ }^{[20-28]}$, which mainly include (E,E)-2,4-decadienal ${ }^{[16]}$, nonaldehyde ${ }^{[20]}$, benzaldehyde $^{[21]}$, phenylacetaldehyde, 2-phenyl-crotonaldehyde ${ }^{[23]}$, $n$-hexanal ${ }^{[24]}$, etc. The grape seed oil, tea seed oil, soybean oil and corn oil contain the highest content of the hexanal. The main volatile component of cold-pressed linseed oil is $n$-hexanal ${ }^{[24]}$. The important aldehydes in sesame paste are phenylacetaldehyde, nonaldehyde, and 2-phenyl-crotonaldehyde, while in the sesame oil are hexanal, (Z)-2-heptenal, (E)-2-octenal and nonanal ${ }^{[15,23]}$. In addition, the major volatile in pressed and solvent-extracted peanut oil is (E,E)-2,4-decadienal ${ }^{[25]}$, and the main aldehydes in rapeseed oil include trans-2-trans-4-heptadiene, aldehyde, trans-2-trans-4-decadienal, and 4-hydroxy-3, 5-dimethoxybenzal dehyde, etc. ${ }^{[26]}$ The relatively high levels of aldehydes in virgin olive oil include (E)-2-hexenal, (E,E)-2,4-heptadienal and nonaldehyde ${ }^{[20]}$. Furthermore, anti-2-hexenal is the main volatile component in European olive oil. The main sources 
of "green" aroma in Spanish olive oil are trans-3-hexenal and cis-3-hexenal ${ }^{[27-28]}$.

\subsection{Furans}

Furans have been detected in the flavors of many roasted oilseeds and their products, which impart fruit flavors, and also have beans and grass flavor in some fats and oils ${ }^{[29]}$. They play the important role in the flavor of hot-processed foods.

Furan compounds are the most important volatile flavors in peanut oil, sesame oil and peanut butter, and also are the main volatiles of perilla seed oil when the roasting temperature is above $170{ }^{\circ} \mathrm{C}^{[5,7,10,30]}$. It was reported that furan compounds are mainly 2-amyl furan ${ }^{[11]}$, 2-ethyl furan ${ }^{[24]}$, etc.

\subsection{Alcohols}

Alcohols were widely found in the volatile flavors of roasted oilseeds and their products. The saturated alcohols have the high thresholds, while the thresholds of unsaturated alcohols are low. Alcohols mainly reflect the fresh, woody and fat flavor with the increase of carbon chain ${ }^{[19]}$.

So far, about 9 alcohols have been reported ${ }^{[6-8]}$. According to the literature, alcohols are the main volatile components of cold-pressed linseed oil and sesame paste ${ }^{[9,24]}$. The alcohols in cold-pressed linseed oil mainly include $n$-hexanol, 2-butanol, 2-methyl butanol and amyl alcohol ${ }^{[24]}$. The sesame paste prepared by stir-frying and baking have the high content of amyl alcohol and 1-hexanol ${ }^{[9]}$. The 1-octen-3-alcohol contributes to flavor of the rapeseed oil, and it appears much more frequently in the first-class rapeseed oil than in the third-grade and fourth-grade rapeseed oil ${ }^{[6]}$.

\subsection{Ketones}

Ketones usually give the sweet and fruity flavor, which endow the roasted oil and their products with a certain fruity flavor $^{[29]}$. The main ketone compounds include 2,3-pentanedione, 2,3-butanedione, 2,3-octanedione ${ }^{[29]}$, etc.

\subsection{Acids}

Acids have less contribution to the overall odor of roasted oilseeds and their products due to their high threshold values ${ }^{[31]}$, though they have the strong fat flavor at high concentrations. The common acids in roasted oilseeds and their products are formic acid, acetic acid, valeric acid, and caproic acid ${ }^{[31]}$. Among them, acetic acid is the main volatile compound in flaxseed oil ${ }^{[32]}$.

\subsection{Esters}

The esters, generally with the low threshold values, have the certain soft effect on the overall odor of the roasted oilseeds and their products. Some esters such as ethylacetate and ethyl hexanoate have an important contribution to the odor of many vegetable oils ${ }^{[19]}$, and the common esters in the roasted oilseeds and their products are cis-3-hexenyl acetate ${ }^{[16]}$.

\subsection{Sulfides}

Sulfides are found in many foods. Dimethyl sulfide has a pleasant crablike flavor at low concentration $(<100 \mu \mathrm{g} / \mathrm{kg})$, but its odor at high concentration is very abnormal ${ }^{[29]}$. Thiazoles usually have clean, nut, vegetable or meat flavor ${ }^{[33]}$.

There are about 4 common sulfur compounds ${ }^{[34-35]}$. Among them, sesame pastes produced by roasting, roasting and steaming contained dimethyl sulfide and dimethyl disulfide ${ }^{[34]}$. Sulfur compounds that distinguished Korean sesame oil from those of other countries included 2,4-dimethylthiazole and 5-ethyl-4-methylthiazole as well as pyrrole compounds ${ }^{[35]}$.

\subsection{Hydrocarbons}

Hydrocarbons generally have the high odor threshold values, and contribute little to the flavor of roasted oilseeds and their products ${ }^{[36]}$. About 30 hydrocarbons have been reported ${ }^{[11,25]}$. Among them, 1-tridecene is a characteristic volatile component of the olive oil ${ }^{[37]}$. Hydrocarbon compounds such as pentane, $n$-heptane, butane, $n$-hexane, and methylcyclopentane are the main volatile components of pressed peanut oil and solvent extracted peanut oil ${ }^{[25]}$.

\section{Formation of Volatile Compounds in Roasted Oilseeds and Their Products}

Flavor are generated mainly through plant secondary metabolism reaction, heat processing and biological fermentation $^{[38]}$. The oilseeds themselves contain components such as oils, proteins, sugars and other components, these compounds undergo a series of reactions during the stir-frying or roasting process to produce a variety of different volatiles which give the special aroma of the roasted oilseeds and their products. Studies to date have shown that there are two major routes for the formation of the volatile flavor components, including biosynthesis by the enzyme's catalytic reaction and chemical conversion ${ }^{[1,6,21]}$. Biosynthesis refers to the formation of volatile flavor substances by a variety of flavor precursors such as fatty acids, pigments, and amino acid glycosides under the action of specific enzymes ${ }^{[6,21,39]}$. Odorants are generated in roasted oilseeds and their products mainly due to chemical conversion routes, such as lipoxygenase processes, amino acid conversion, lipid oxidation, Maillard reaction and caramelization reaction, etc. ${ }^{[1,20-21,40]}$ The chemical pathways are discussed in detail as the follows.

\subsection{Maillard Reaction}

Maillard reaction is a kind of non-enzymatic browning reaction between amino compounds (such as amines, amino acids, protein, etc.) and carbonyl compounds (such as reducing sugar, lipids, and the resulting aldehydes, ketones, etc.), and widely exists in food processing. It is one of the most important ways to produce flavor components in roasted oilseeds and their products $^{[38]}$. In addition, the thermal degradation products of oils during roasting can react with a small amount of proteins and amino acids to form the flavor and color of oils ${ }^{[1,6,20]}$, such as pyrazine, furan, pyridine and pyrrole, thiophene, thiazole, thiophene, etc. $[21,40]$

Maillard reaction is divided into three steps. The primary step is condensation reaction between the reducing sugar and the amino-group, and formation of the Shiff base by loss of one molecule of water, then formation of a glucosyl amine, 1-amino- 
1-deoxy-2-ketose and 1-aldose-2-amino-2-deoxy intermediates. Intermediate step is formation of the reduced ketones, furfural and unsaturated carbonyl compounds by the reducing ketone route, the reducing glucuronone and furfural pathway, and the Strecker degradation route. Final step involves polymerization between aldehydes and amines.

In the intermediate steps, a large number of active intermediates are formed, which can be further condensed, polymerized, or reacted with amino acids to form flavor compounds such as high molecular pigments, pyrazine, and imidazolium rings ${ }^{[41]}$. After a complicated process, the Maillard reaction finally produces a large number of nitrogen-oxygen heterocyclic compounds, such as pyrazine, pyridine, pyrrole, furfural, furanone, etc. These compounds usually have a $\mathrm{C}_{5}-\mathrm{C}_{10}$ alkyl substituent, which are usually derived from the aliphatic aldehyde, and $\mathrm{N}$ is mainly derived from amino acids in food ${ }^{[42]}$. These heterocyclic compounds have an important contribution to the roasted, roasted and nutty flavors of roasted foods ${ }^{[43]}$.

\subsection{Lipid Oxidiation}

Lipid oxidation is one of the main sources of oil flavor. Most of the flavor compounds in oils are produced by the reaction of glycerol triacylglycerol and unsaturated fatty acids in polar lipids with oxygen ${ }^{[4]}$. Frying seeds at high temperature for a long time creates enough conditions for the oxidative degradation of fatty acids.

Auto-oxidation of fatty acids in vegetable oils, especially linoleic acid or $\alpha$-linoleic acid, can form unique flavor substances through a series of oxidation reactions. The oxidation of fatty acids is a free-radical chain reaction. The hydroperoxides of unsaturated fatty acids are further degraded to produce alkoxy radicals and hydroxyl radicals. Finally, alkoxy radicals are degraded to form alcohols, aldehydes, ketones, alkanes, etc. ${ }^{[44-45]}$ Aldehydes are the most important flavor compounds produced by the oxidative decomposition of fats. The flavor of aldehydes is usually described as grass flavor, paint flavor, etc. [43]

\subsection{Lipoxygenase and Amino Acid Conversion Processe}

Lipoxygenase processes is the key route for the green odor notes of virgin olive oil aroma, in which polyunsaturated fatty acids are gradually converted into aldehydes and alcohols under the concerted action of lipoxygenase, isomerase, acyl hydrolase, and hydroperoxidelyase ${ }^{[46]}$. Among them, the lipoxygenase (LOX) pathway enzymatically produced $\mathrm{C}_{6}$ and $\mathrm{C}_{5}$ compounds via polyunsaturated fatty acids, and $\mathrm{C}_{5}$ compounds are contributors to some green sensory perceptions ${ }^{[47]}$. In particular, the $\mathrm{C}_{6}$ straight-chain saturated and unsaturated aldehydes represent the most important part of the volatile compounds in high quality virgin olive oil ${ }^{[48]}$.

Amino acids have been reported to be the important precursors for flavor formation, and they can also produce flavor compounds, such as 2-Methylthiazoline, $\alpha$-Picoline, 2-Methylthiophene, etc. These flavor compounds produced by thermal degradation of sulfur-containing amino acids, and proline and leucine can be converted into branched alcohols and esters $^{[49-50]}$.

\section{Factors Affecting the Volatile Flavor Components}

The volatile flavor substances of the roasted oilseeds and their products are influenced by the various factors such as variety, origin, fruit maturity, refining, processing, etc. during the formation process ${ }^{[6,21]}$.

\subsection{Impact of Oilseed Varieties}

The volatile flavor substances produced by different oil crops have great differences in composition and content, and each plant oil contains unique volatile flavor substances. For example, sesame oil has a roasted sesame flavor, rapeseed oil has a pungent smell of grass, and olive oil has an olive fruit flavor. Studies have shown that varieties are the main factors affecting the formation of olive oil aroma. Examining the differences in the contents of hexaldehyde, ethanol, and 3-hexenyl acetate can distinguish between different varieties of olive oils ${ }^{[42,51-54]}$.

\subsection{Impact of Oilseeds Production Areas}

Oil crops in different geographical locations have different environmental factors, such as rainfall and sunshine hours, which play an important role in the formation of volatile flavoring substances in roasted oilseeds and their products.

Geographical factors have a strong effect on the formation of volatile substances of camellia oil and virgin olive oil ${ }^{[20-21]}$. According to the research, the tea seed oil from Qingtian origin has the stronger grass flavor, and that from Jiande origin has the higher scores of nut, sweet note ${ }^{[21]}$. The mass fraction of (E)-2-hexenal in Chinese olive oil is higher, while the relative mass fractions of Caproicacidhexneylester and (E)-2-dodecene in Spanish extra virgin olive oil is higher ${ }^{[20]}$.

\subsection{Impact of Processing Factors}

The foods that are roasted or involved in the extended heat treatment in their preparation typically form the volatile flavor compounds, and the key factors involved are usually the temperature and time of roasting. Studies have shown that pyridazine, furan and other heterocyclic substances in oilseeds will rise with the increase of roasting temperature ${ }^{[11,13,21]}$.

\subsubsection{Effects of Processing Factors on Volatile Flavor of} Sunflower Oil

Studies showed that pyrazine and other heterocyclic compounds in sunflower oil were increased after roasting ${ }^{[12-13,55]}$.

The characteristic flavor component of sunflower oil is most obvious in the roasting conditions of $140{ }^{\circ} \mathrm{C} / 30 \mathrm{~min}$ and $150{ }^{\circ} \mathrm{C} / 20 \mathrm{~min}$, under which the species and content of pyrazine compounds account for the largest proportion ${ }^{[50]}$. The volatile substances in cold-pressed sunflower oil are mainly terpenes, alkanes, and aldehydes and ketones, and the main volatile substances in sunflower seeds with hot-fried seeds below $150{ }^{\circ} \mathrm{C}$ are similar to the cold-pressed sunflower oil, but there are the differences in the contents. When the frying temperature is above $170{ }^{\circ} \mathrm{C}$, the main volatile substances are pyrazine and other heterocyclic substances, aldehydes and ketones ${ }^{[12]}$. The sunflower oil obtained by pressing and roasting has more volatile components and better flavor ${ }^{[56]}$, and microwaves also 
have a significant effect on the quality of sunflower oil ${ }^{[57]}$. There are a variety of aldehydes and esters compounds in the volatile components of sunflower seeds. A large number of pyrazine compounds are produced in sunflower seeds after roasting, and the contents of unsaturated aldehydes are also increase ${ }^{[13]}$.

\subsubsection{Effects of Processing Factors on Volatile Flavor of Rapeseed Oil}

According to the literature, the processing technology has a significant effect on the flavor of rapeseed oil ${ }^{[26]}$. With the increase of roasting temperature, the relative contents of glucosinolate degradation products in rapeseed oil are decreased, the content of heterocyclic compounds and oxidized volatiles are increased, and the heterocyclic compounds are mainly pyrazine compounds ${ }^{[4,14,58]}$.

Rapeseed oil can produce the best flavor by frying at $115^{\circ} \mathrm{C}$, and the relative contents of glucosinolate degradation products in rapeseed oil are decreased with the increase of frying temperature. Pyrazines are the main heterocyclic substances in rapeseed oil, which have the highest relative content at $120^{\circ} \mathrm{C}^{[14]}$. Moreover, the kinds and relative contents of heterocyclic compounds in pre-pressed oil obtained by high-temperature steaming and pressing are significantly higher than other rapeseed oils, and the oxidation volatiles in first-grade oil were increased, but the degradation products of glucosinolates are not detected $^{[26]}$. Compared with rapeseed raw oil, the species and relative contents of glucosinolates and heterocyclic substances in refined rapeseed oil are significantly decreased, while aldehydes and other oxidizing volatiles are significantly increased ${ }^{[59]}$.

\subsubsection{Effects of Processing Factors on Volatile Flavor of Peanut Oil}

The flavor characteristics of the peanut oil is very similar during the initial roasting time of $30 \mathrm{~min}$, while it is significantly different after $30 \mathrm{~min}$. With elongation the roasting time, the contents of nitrogen-containing heterocyclic compounds increases rapidly, and the nitrogen-containing heterocyclic compounds become the main flavor substance in peanut oil in place of the non-heterocyclic compounds ${ }^{[60]}$. In addition, the pyrazines and pyrrolics are found in the volatile components of pressed peanut oil, but the pyrazines are not identified in the solvent extracted peanut oil ${ }^{[25]}$. Besides, the volatile components of fresh peanuts are mainly alkanes, alcohols, aldehydes, and aromatic benzene, while roasted or stir-fried peanuts contain mainly pyrazines hydrocarbons, aldehydes, ketones, and esters ${ }^{[61-62]}$. Moreover, peanuts produce the bad flavor such as cardboard, stale and bitterness flavor after microwave treatment and the worst flavor is produced through microwave treatment for $11 \mathrm{~min}^{[61]}$. These odorous substances are related to some compounds such as ethanol, acetaldehyde, and ethyl acetate, and the determined odor substances are phenylacetaldehyde and guaiacol ${ }^{[63-64]}$.

\subsubsection{Effects of Processing Factors on Volatile Flavor of Sesame Oil}

Studies have shown that the roasting temperature has a great influence on the flavor component of sesame oil than the roasting time. When the temperature is too low, it is not conducive to the formation of flavor substances in sesame oil, while too high temperature will destroy the flavor components of sesame oil ${ }^{[65-67]}$. When the roasting temperature is between $213{ }^{\circ} \mathrm{C}$ and $247{ }^{\circ} \mathrm{C}$, the changes of total volatile compounds and pyrazine contents of sesame oil are more obvious, and sesame oil obtained by roasting sesame seeds at $213{ }^{\circ} \mathrm{C}$ and $230{ }^{\circ} \mathrm{C}$ is obviously different from those processed at other roasting temperatures ${ }^{[66,68]}$. Moreover, the recovery of total volatiles components of sesame oil increase with the roasted degree (temperature and time), and the amount of pyrazines reach the maximum after roasting at $200{ }^{\circ} \mathrm{C}$ for $30 \mathrm{~min}$. On the contrary, the relative content of monoalkylpyrazine in the deep roasted sesame oil is decreased with the degree of roasting, but thiazoles, guaiacol, and 2-furanmethylthiol are increased ${ }^{[69-70]}$.

\subsubsection{Effects of Processing Factors on Volatile Flavors of Other Roasting Oilseeds and Their Products}

The volatile flavor substances of sesame paste under stir-frying, roasting and microwaving are mainly pyrazines, aldehydes, furans, alcohols and hydrocarbons. Among these, the pyrazines are up to $31.09 \%$ in the volatiles of the sesame paste by microwaving, and the aldehydes are $14.97 \%$ and $24.36 \%$ in sesame paste by stir-frying and roasting, respectively. The content of aldehydes in the stir-frying sesame paste are as high as $17.17 \%$, and the content of alcohols in three processing methods are greater than $30 \%{ }^{[8,71]}$.

The cold-pressed linseed oil has the highest content of alcohols. Compared with the cold-pressed linseed oil, the relative contents of alcohols in the hot-pressed linseed oil are decreased, but the relative content of aldehydes and heterocyclic substances are increased rapidly ${ }^{[24]}$.

The perilla seed oil has a relatively high content of aldehydes when it is roasted at $170{ }^{\circ} \mathrm{C}$, and when the roasting temperature is above $170{ }^{\circ} \mathrm{C}$, the main volatiles of perilla seed oil are pyrazine and furan. In all roasted perilla seed oils, the contents of perilla aldehyde and perilla ketone remain almost unchanged, which could be used to distinguish perilla seed oil from other roasted vegetable seed oil ${ }^{[5]}$.

The characteristic aroma substances of the roasted tea seed oil are mainly related to fermentation, alcohols, ketones and heterocyclics, and the roasting conditions have a significant influence on the formation of the characteristic aroma of tea seed oil. The total contents of aldehydes greatly fluctuate with the degree of roasting, and the alcohols overall show a downward trend. On the contrary, the contents of heterocyclics increase significantly with the degree of roasting ${ }^{[21]}$.

\section{Conclusions and Prospects}

There are more work to do on the volatile flavor research of roasted oil seeds and their products, and especially in sesame paste and peanut butter, etc. The more efficient and convenient detection methods are need to be developed. In addition, the formation mechanism of flavor substances in roasted oil seeds and their products needs to be further studied. Studying the volatile flavor substances is helpful to the development of new 
products and the techniques for the detection of adulteration.

\section{Conflict of Interest}

The authors declare that there is no conflict of interest.

\section{References}

[1] ZHAO F F. Research on the detection technology of oil volatiles in oil[D]. Beijing: Chinese Academy of Agricultural Sciences, 2012.

[2] WEN Z Y, SUN B G, LIANG M L, et al. Lipid oxidation to produce flavor substances[J]. China Oils and Fats, 2004(9): 41-44

[3] LIU J H, REN H L. Changes of trace components in the processing of vegetable oils[J]. China Oils and Fats, 2000, 25(4) 49-52.

[4] GRACKA A, JELEN H H, MAJCHER M, et al. Flavoromics approach in monitoring changes in volatile compounds of virgin rapeseed oil caused by seed roasting[J]. Journal of Chromatography A, 2015, 1428: 292-304.

[5] KIM S J, YOON H N, RHEE J S. The effects of roasting temperatures on the formation of headspace volatile compounds in perilla seed oil[J]. Journal of the American Oil Chemists Society, 2000, 77(4): 451-456.

[6] DENG L. Study on the characteristics of aroma components and nutrient compositions of rapeseed oil[D]. Nanchang: Nanchang University, 2017.

[7] LOU F, LIU Y, SUN X H, et al. Identification of volatile flavor components in peanut butter[J]. Food Science, 2009, 30(24): 393-396.

[8] ZHANG M, LIU G Q, JIA H F, et al. Different flavors of different hot processing sesame pastes[J]. Food Industry Science and Technology, 2018, 39(10): 262-266.

[9] YAN Q. Study on roasting sesame aroma components[J]. Food Industry, 2014, 35(3): 245-247.

[10] LIU X J. Effects of fried seeds on the flavor and quality of peanut oil[D]. Wuxi: Jiangnan University, 2011.

[11] ZHOU P P, HUANG J H, SONG Z H, et al. Identification of volatile flavor components of sunflower sunflower seed oil[J]. Food Industry Science and Technology, 2012, 33(14): 128-131.

[12] HONG Z T, CHEN J, FAN L, et al. Analysis of volatile substances in cold and hot pressed sunflower oil by HS-SPME-GC-MS[J]. China Oils and Fats, 2015, 40(2): 90-94.

[13] JIA C X, MAO D B, SUN X L, et al. Analysis and comparison of chemical composition of sunflower seeds before and after baking[J]. Food Research and Development, 2008(7): 111-115.

[14] WU L, XU L, XIE J, et al. Effects of different stir-frying temperature on volatile flavor compounds of rapeseed oil[J]. China Oils and Fats, 2012, 37(11): 39-43.

[15] TANG X D, QIN Z, YANG Y, et al. Volatile flavor components in sesame oil of different aroma types[J]. China Oils and Fats, 2013 , 38(6): 87-90.

[16] YANG C Y, LIU X M, WANG S Y, et al. Analysis of volatile flavor components of vegetable oil by SPME-GC/MS[J]. Journal of the Chinese Cereals and Oils Association, 2015, 30(10): 127-134.

[17] LI S R, WANG L, ZHANG C H, et al. Study on the key flavor compounds in baking peanut[J]. Chinese Agricultural Science, 2010, 43(15): 3199-3203

[18] WANG Y F. Study on volatile flavor components of sesame oil with different raw materials[J]. Cereal and Food Industry, 2017, 24(2): 24-27.

[19] XU X. Study on the changes of fatty acids and volatile components in the oxidation of vegetable oils[D]. Hangzhou: Zhejiang Gongshang University, 2013.

[20] ZHONG C, XUE Y L, WANG X G, et al. Research progress on flavor compounds of virgin olive oil[J]. China Oils and Fats, 2013 , 38(8): 89-92.

[21] LIN L. Aroma quality analysis of camellia oil from different sources[D]. Shanghai: Shanghai University of Technology, 2016.

[22] IVANOVA-PETROPULOS V, MITREV S, STAFILOV T, et al.
Characterisation of traditional Macedonian edible oils by their fatty acid composition and their volatile compounds[J]. Food Research International, 2015, 77(3): 506-514.

[23] SUN Q, WANG J B, ZHANG L X, et al. Effects of pretreatment process on flavor components of sesame sauce $[\mathrm{J}]$. Food Industry Science and Technology, 2017, 38(24): 6-9.

[24] YANG J E, HUANG Q D, ZHOU Q, et al. Comparison of volatile components in flaxseed oil from cold pressed and hot pressed[J] Chinese Journal of Oil Crop Sciences, 2013, 35(3): 321-325.

[25] WANG Y. Study on flavor components and unsaponifiables of fragrant Peanut Oil[D]. Zhengzhou: Henan University of Technology, 2011.

[26] YANG M, LIU C S, ZHOU Q, et al. Effect of processing technology on volatile components of rapeseed oil[J]. Chinese Journal of Oil Crop Sciences, 2010, 32(4): 551-557.

[27] SUN S M, XIE Y L, ZHAO W H, et al. Research progress on characteristic chemical constituents and flavor substances of olive oil[J]. Journal of Henan University of Technology: Natural Science Edition, 2015, 36(5): 113-119.

[28] AND R A, MORALES M T. Characterization of olive ripeness by green aroma compounds of virgin olive oil[J]. Journal of Agricultural \& Food Chemistry, 1998, 46(3): 1116-1122.

[29] QIU H Q, CHEN D, LIU W H. Study on volatile components of rapeseed oil by solvent distillation gas chromatography-mass spectrometry[J]. Zhejiang Chemical Industry, 2009, 40(1): 21-23.

[30] LIU P N. Study on the volatile flavor components of sesame oil[J]. Journal of the Chinese Cereals and Oils Association, 2005(6): 88-90.

[31] MASON M E, JOHNSON B, HAMMING M C. Volatile components of roasted peanuts. Major monocarbonyls and some noncarbonyl components[J]. Journal of Agricultural \& Food Chemistry, 1967, 15(1): 63-73.

[32] KRIST S, STUEBIGER G, BAIL S, et al. Analysis of volatile compounds and triacylglycerol composition of fatty seed oil gained from flax and false flax[J]. European Journal of Lipid Science \& Technology, 2010, 108(1): 48-60.

[33] YURCHENKO S, MOLDER U. Volatile N-Nitrosamines in various fish products[J]. Food Chemistry, 2006, 96(2): 325-333.

[34] SHAHIDI F, AISHIMA T, ABOU-HARBIA H A, et al. Effect of processing on flavor precursor amino acids and volatiles of sesame paste (Tehina) [J]. Journal of the American Oil Chemists Society, 1997, 74(6): 667.

[35] SHIN B R, SONG H W, LEE J G, et al. Comparison of the contents of benzo (a) pyrene, sesamol and sesamolin, and volatiles in sesame oils according to origins of sesame seeds[J]. Applied Biological Chemistry, 2016, 59(1): 129-141.

[36] WANG Y T, WANG S F, LIU M Z, et al. Application of HS-SPME and GC/MS techniques for detecting flavor substances in feeding pig muscles[J]. Journal of Nuclear Agriculture, 2008, 22(5): 654-660.

[37] ZHUANG X L. The application of chemometrics in the analysis of olive oil quality and identification of wheat[D]. Beijing: Capital Normal University, 2008.

[38] DING N. Food Flavor Chemistry[M]. Beijing: China Light Industry Press, 1996: 144-145.

[39] ZHU G B, LU H J. Food Flavor Principle and Technology[M]. Beijing: Peking University Press, 1996: 93-123.

[40] ZHOU R B. The flavor components of sesame oil[J]. Journal of the Chinese Cereals and Oils, 2006, 21(3): 310-315.

[41] WU S, QIN J. Mechanism of Maillard Reaction[J]. Journal of Guizhou University of Technology: Natural Science Edition, 2005, 34(4): 17-20.

[42] WONG J M, BERNHARD R A. Effect of nitrogen source on pyrazine formation[J]. Journal of Agricultural and Food Chemistry, 1988, 36(1): 123-129.

[43] FEREIDOON S. Bailey's Industrial Oil and Fat Products[M]. 6th ed., New York: John Wiley \& Sons, Inc., 2005.

[44] MARIA E E. Olive oil aroma evaluation by gas chromatographic method: A critical review[J]. Critical Reviews in Analytical 
Chemistry, 2011, 41(1): 70-80.

[45] DRUMM T D, SPANIER A M. Changes in the content of lipid autoxidation and sulfur-containing compounds in cooked beef during storage $[\mathrm{J}]$. Journal of Agricultural \& Food Chemistry, 1991, 39(2): 336-343.

[46] OLIAS J M, PEREZ A G, RIOS J J, et al. Aroma of virgin olive oil: Biogenesis of the "green" odor notes[J]. Journal of Agricultural and Food Chemistry, 1993, 41(12): 2368-2373.

[47] ANGEROSA F, MOSTALLINO R, BASTI C, et al. Virgin olive oil odour notes: their relationships with volatile compounds from the lipoxygenase pathway and secoiridoid compounds[J]. Food Chemistry, 2000, 68(3): 283-287.

[48] ANGEROSA F, SERVILI M, SELVAGGINI R, et al. Volatile compounds in virgin olive oil: occurrence and their relationship with the quality[J]. Journal of Chromatography A, 2004, 1054(1): 17-31.

[49] KATO S, KURATA T, ISHIGURO S, et al. Additional volatile compounds produced by pyrolysis of sulfur-containing amino acids[J]. Journal of the Agricultural Chemical Society of Japan, 1973, 37(7): 1759-1761.

[50] TRESSLR, DRAWERT F. Biogenesis of banana volatiles[J] Journal of Agricultural \& Food Chemistry, 1973, 21(4): 560-565

[51] SHEN X L, LI C. Lipids and meat flavor[J]. Meat Research, 2008 (3): $25-28$.

[52] ARINÃ K, ELMACI Y. Aroma characterization of virgin olive oil from two turkish olive varieties by SPME/GC/MS[J] International Journal of Food Properties, 2011, 14(5): 1160-1169.

[53] YAO J N, WANG D P, PAN W D, et al. Analysis of aroma components of tea oil produced by wolfberry in SPME-GCMS[J] Science and Technology of Cereals, Oils and Foods, 2014, 22(2): 64-66.

[54] RANALLI A, CONTENTO S, SCHIAVONE C, et al. Malaxing temperature affects volatile and phenol composition as well as other analytical features of virgin olive oil[J]. European Journal of Lipid Science \& Technology, 2015, 103(4): 228-238.

[55] ZHOU P P. effect of sunflower seed baking on flavor and quality of sunflower seed oil[D]. Wuxi: Jiangnan University, 2013.

[56] CHAI J, JIN Q Z, XUE Y L, et al. Effect of oil production process on the quality of sunflower oil[J]. China Oils and Fats, 2016 , 41(4): 56-61.

[57] CHEN J, HONG Z T, LIU G Q, et al. Effects of microwave roasting on the quality and volatile substances of sunflower seed oil[J]. Modern Food Science and Technology, 2015, 31(8): 211-218.

[58] WEI F, YANG M, ZHOU Q, et al. Varietal and processing effects on the volatile profile of rapeseed oils[J]. LWT-Food Science and Technology, 2012, 48(2): 323-329.

[59] XIE Q, XU L, WU L, et al. SPME-GC-MS analysis of volatile flavor components of rapeseed oil and refined rapeseed oil[J]. China Oils and Fats, 2012, 37(8): 84-87.

[60] LIU X J. Effects of fried seeds on the flavor and quality of peanut oil[D]. Wuxi: Jiangnan University, 2011.

[61] SHI W Q. Identification of authentic components of volatile odor of peanut and peanut oil[D]. Wuhan: Wuhan Polytechnic University, 2012.

[62] CHETSCHIK I, GRANVOGL M, SCHIEBERLE P. Quantitation of key peanut aroma compounds in raw peanuts and pan-roasted peanut meal. Aroma reconstitution and comparison with commercial peanut products[J]. Journal of Agricultural \& Food Chemistry, 2010, 58(20): 11018-11026.

[63] LIN M, LU J W, MA T J, et al. Research progress on volatile flavor substances in peanut $[\mathrm{J}]$. Food Research and Development, 2013, (12): 116-110.

[64] SCHIRACK A V, DRAKE M A, SANDERS $\mathrm{T} H$, et al Characterization of aroma-active compounds in microwave blanched peanuts[J]. Journal of Food Science, 2010, 71(9): C513-C520.

[65] CONG S. Effect of roasting process on flavor and quality of sesame oil in water[D]. Zhengzhou: Henan University of Technology, 2013.

[66] MIN H P, MIN K J, YEO J D, et al. Application of solid phase-microextraction (spme) and electronic nose techniques to differentiate volatiles of sesame oils prepared with diverse roasting conditions[J]. Journal of Food Science, 2011, 76(1): C80-C88.

[67] SONG G X, ZHANG Y, CHENY H. Study on volatile flavor components in fragrant sesame oil and sesame oil[J]. Cereal and Food Industry, 2015, 22(3): 10-13.

[68] QIN Z. Analysis of sesame oil flavors[D]. Zhengzhou: Zhengzhou University, 2012.

[69] SU N R, KIM S M, XI J, et al. Influence of seed roasting process on the changes in volatile compounds of the sesame (sesamum indicum 1.) oil[M]//. Shahidi F, Ho C T. Flavor Chemistry of Ethnic Foods. Switzerland: Springer, 1999: 229-237.

[70] SHIMODA M, NAKADA Y, NAKASHIMA M, et al. Quantitative comparison of volatile flavor compounds in deep-roasted and light-roasted sesame seed oil[J]. Journal of Agricultural and Food Chemistry, 1997, 45(8): 3193-3196.

[71] SUN Q, WANG J B, ZHANG L X, et al. Effect of pretreatment process on flavor matter of sesame paste[J]. Science \& Technology of Food Industry, 2017 (24): 6-9. 